\title{
INEQUALITIES RELATED WITH CARLSON'S INEQUALITY
}

\author{
SORINA BARZA AND EMIL C. POPA
}

\begin{abstract}
We prove a generalization of Carlson's inequality. We improve a previous equivalent inequality of Carlson's inequality and give some examples.
\end{abstract}

\section{Introduction}

Let $\left\{a^{n}\right\}, n=1,2, \ldots$ be a sequence of nonnegative numbers and $f$ a measurable function on $[0, \infty]$. In 1934, Carlson [4] proved that the somewhat curious inequalities

$$
\sum_{n=1}^{\infty} a_{n}<\sqrt{\pi}\left(\sum_{n=1}^{\infty} a_{n}^{2}\right)^{\frac{1}{4}}\left(\sum_{n=1}^{\infty} n^{2} a_{n}^{2}\right)^{\frac{1}{4}}
$$

and

$$
\int_{0}^{\infty}|f(x)| d x \leq \sqrt{\pi}\left(\int_{0}^{\infty} f^{2}(x) d x\right)^{\frac{1}{4}}\left(\int_{0}^{\infty} x^{2} f^{2}(x) d x\right)^{\frac{1}{4}}
$$

hold and $C=\sqrt{\pi}$ is the best possible in both cases.

In 1936 Hardy [6] presented two simpler proots of (1) and (2). He has also showed there that the inquality

$$
(f(0))^{4} \leq 4 \int_{0}^{\infty} f^{2}(x) d x \int_{0}^{\infty} f^{\prime 2}(x) d x
$$

is equivalent with (2). We have equality in (3) when $f$ is an exponential.The inequality (3) is also given as an exercise in variational methods in [HLP, page 194(Th.263)]. In particular Hardy observed that (1) follows in fact from Schwarz inequality $\sum x_{n} y_{n} \leq$ $\left(\sum x_{n}^{2}\right)^{\frac{1}{2}}\left(\sum y_{n}^{2}\right)^{\frac{1}{2}}$ apllied to the sequences $x_{n}=a_{n}\left(\alpha+\beta n^{2}\right)^{\frac{1}{2}}$ and $y_{n}=\left(\alpha+\beta n^{2}\right)^{\frac{1}{2}}$. We remark here that (1),(2) and their generalizations have application to some moment problems (see [8]), in the interpolation theory (see [5],[9],[10]) and optimal reconstruction of a sampling signal (see [3]).

The aim of this paper is give further generalizations and related related results of (2) and (3).The main results (Theorems $2.1,2.3,2.4$ ) are stated and proved in Section 2. In Section 3 we prove other results and present some conclusions and remarks.

Received May 6, 1997.

1991 Mathematics Subject Classification. 26D15, 26D10. 


\section{Integral Inequalities Related with Carlson's Inequality}

Theorem 2.1. Let $f, g:[0, \infty] \rightarrow \mathbb{R}$ be Lebesgue measurable funtions and $g$ bc differentiable. If $g(0)=0$, $\lim _{x \rightarrow \infty} g(x)=\infty$ and $0<m=\inf _{x \in[0, \infty)} g^{\prime}(x)<\infty$, then

$$
\left(\int_{0}^{\infty} f(x) d x\right)^{4} \leq \frac{\pi^{2}}{m^{2}} \int_{0}^{\infty} f^{2}(x) d x \int_{0}^{\infty} g^{2}(x) f^{2}(x) d x
$$

Proof. Let $\lambda>0$ and let, $S=\int_{0}^{\infty} f^{2}(X) d x$ and $T=\int_{0}^{\infty} g^{2}(x) f^{2}(x) d x$.

If $S=\infty$ or $T=\infty$, then (4) holds trivially so we may without loss of generality assume that $S<\infty$ and $T<\infty$. By using the Schwarz inequality and elementary calculations we find that

$$
\begin{aligned}
\left(\int_{0}^{\infty} f(x) d(x)\right)^{2} & =\left(\int_{0}^{\infty} f(x) \sqrt{\frac{\lambda+\frac{1}{\lambda} g^{2}(x)}{g^{\prime}(x)}} \sqrt{\frac{g^{\prime}(x)}{\lambda+\frac{1}{\lambda} g(x)^{2}}} d(x)\right)^{2} \\
& \leq \int_{0}^{\infty} \frac{\lambda+\frac{1}{\lambda} g^{2}(x)}{g^{\prime}(x)} f^{2}(x) d x \int_{0}^{\infty} \frac{g^{\prime}(x) d x}{\lambda+\frac{1}{\lambda} g^{2}(x)} \\
& \leq\left.\frac{1}{m}\left(\lambda S+\frac{1}{\lambda} T\right) \arctan \frac{g(x)}{\lambda}\right|_{0} ^{\infty}=\frac{\pi}{2 m}\left(\lambda S+\frac{1}{\lambda} T\right)
\end{aligned}
$$

By choosing $\lambda=\sqrt{\frac{T}{S}}$ we obtain that

$$
\left(\int_{0}^{\infty} f(x) d x\right)^{2} \leq \frac{\pi}{m} \sqrt{S T}
$$

and (4) is proved.

Remark 2.2. The inequality (4) is a generalization of Carlson inequality (2). We get it just by taking $g(x)=x$. Then

Theorem 2.3. Let $f:[0,1] \rightarrow \mathbb{R}$ be Lebesgue measurable function and $p, q \in[0,2)$.

$$
\left(\int_{0}^{1} f(x) d x\right)^{4} \leq B^{2}\left(\frac{2-p}{2}, \frac{2-q}{2}\right) \int_{0}^{1} x^{p} f^{2}(x) d x \int_{0}^{1}(1-x)^{q} f^{2}(x) d x
$$

where $B\left(\frac{2-p}{2}, \frac{2-q}{2}\right)$ is the Euler beta function.

Proof. Let $\lambda>0$ and let $S=\int_{0}^{1} x^{p} f^{2}(x) d x$ and $T=\int_{0}^{1}(1-x)^{q} f^{2}(x) d x$.

If $S=\infty$ or $T=\infty$, then (5) holds trivially so we may without loss of generality assume that $S<\infty$ and $T<\infty$. By using the Schwarz inequality and elementary calculations we find that

$$
\left(\int_{0}^{1} f(x) d x\right)^{2}=\left(\int_{0}^{1} f(x) \sqrt{\lambda x^{p}+\frac{1}{\lambda}(1-x)^{q}} \frac{1}{\sqrt{\lambda x^{p}+\frac{1}{\lambda}(1-x)^{q}}} d x\right)^{2}
$$




$$
\begin{aligned}
& \leq \int_{0}^{1}\left(\lambda x^{p}+\frac{1}{\lambda}(1-x)^{q}\right) f^{2}(x) d x \int_{0}^{1} \frac{d x}{\lambda x^{p}+\frac{1}{\lambda}(1-x)^{q}} \\
& =\left(\lambda S+\frac{1}{\lambda} T\right) \int_{0}^{1} \frac{d x}{\lambda x^{p}+\frac{1}{\lambda}(1-x)^{q}}
\end{aligned}
$$

By arithmethic mean - geometric mean inequlity we get

$$
\lambda x^{p}+\frac{1}{\lambda}(1-x)^{q} \leq 2 x^{p / 2}(1-x)^{q / 2}, \quad x \in[0,1]
$$

i.e.

$$
\frac{1}{\lambda x^{p}+\frac{1}{\lambda}(1-x)^{q}} \leq \frac{1}{2} x^{-p / 2}(1-x)^{-q / 2}, \quad x \in(0,1)
$$

Thus

$$
\int_{0}^{1} \frac{d x}{\lambda x^{p}+\frac{1}{\lambda}(1-x)^{q}} \leq \frac{1}{2} B\left(\frac{2-p}{2}, \frac{2-q}{2}\right)
$$

and

$$
\left(\int_{0}^{1} f(x) d x\right)^{2} \leq \frac{1}{2} B\left(\frac{2-p}{2}, \frac{2-q}{2}\left(\lambda S+\frac{1}{\lambda} T\right) .\right.
$$

For $\lambda=\sqrt{\frac{T}{S}}$ we obtain (5).

The next theorem is somehow an improvement of inequality (3).

Theorem 2.4. Let $f:[0,1] \rightarrow \mathbb{R}$ be differentiable, with its derivative integrable and

$$
C|f(0)| \leq \int_{0}^{1} f(x) d x
$$

where $C>1$. Then

$$
f^{4}(0) \leq \frac{1}{C^{2}(C-1)^{2}} \int_{0}^{1} f^{2}(x) d x \int_{0}^{1} f^{\prime 2}(x) d x .
$$

Proof. By hypotesis and Schwarz inequality we have

$$
f^{2}(0) \leq \frac{1}{C^{2}} \int_{0}^{1} f^{2}(x) d x .
$$

On the other hand

$$
|f(t)-f(0)|=\left|\int_{0}^{t} f^{\prime}(x) d x\right| \leq \int_{0}^{1}\left|f^{\prime}(x)\right| d x \leq\left(\int_{0}^{1} f^{\prime 2}(x) d x\right)^{1 / 2}
$$

for $t \in[0,1]$. Intergrating on $[0,1]$ we get

$$
\int_{0}^{1}|f(t)-f(0)| d t \leq\left(\int_{0}^{1} f^{\prime 2}(x) d x\right)^{1 / 2}
$$


But by hypotesis we have

$$
\begin{aligned}
(C-1)|f(0)| & \leq \int_{0}^{1} f(t) d t-|f(0)| \leq\left|\int_{0}^{1} f(t) d t\right|-|f(0)| \\
& \leq\left|\int_{0}^{1}(f(t)-f(0)) d t\right| \leq \int_{0}^{1}|f(t)-f(0)| d t .
\end{aligned}
$$

From (8) and (9) we have

$$
f^{2}(0) \leq \frac{1}{(C-1)^{2}} \int_{0}^{1} f^{\prime 2}(x) d x
$$

(7) and (10) give (6) and the proof is over.

\section{Final Conclusions, Examples and Remarks}

Corollary 3.1. If $f$ satysfies the conditions of Theorem 2.4 and is an increasing function on $[0,1]$ and $C_{1}>0$, then

$$
f^{4}(0) \leq \frac{1}{C^{2}(C-1)^{2} C_{1}^{2}}\left(\int_{0}^{1}\left(\left(x-\frac{1}{2}\right)^{2 n+1}+C_{1}\right) f(x) d x\right)^{2} \int_{0}^{1} f^{\prime 2}(x) d x
$$

where $n \in \mathbb{N}$.

Proof. Becasue

$$
\int_{0}^{1}\left(x-\frac{1}{2}\right)^{2 n+1} f(x) d x=\int_{0}^{1}\left(\frac{1}{2}-x\right)^{2 n+1} f(1-x) d x
$$

we get

$$
\begin{aligned}
& 2 \int_{0}^{1}\left(x-\frac{1}{2}\right)^{2 n+1} f(x) d x=\int_{0}^{1}\left(x-\frac{1}{2}\right)^{2 n+1}(f(x)-f(1-x)) d x \\
& =\int_{0}^{1 / 2}\left(x-\frac{1}{2}\right)^{2 n+1}(f(x)-f(1-x)) d x+\int_{1 / 2}^{1}\left(x-\frac{1}{2}\right)^{2 n+1}(f(x)-f(1-x)) d x .
\end{aligned}
$$

Since $f$ is increasing on $[0,1]$ it is easy to see that

$$
\int_{0}^{1}\left(x-\frac{1}{2}\right)^{2 n+1} f(x) d x \geq 0
$$

and then

Hence

$$
\int_{0}^{1}\left(\left(x-\frac{1}{2}\right)^{2 n+1}+C_{1}\right) f(x) d x \geq C_{1} \int_{0}^{1} f(x) d x
$$

$$
|f(0)| \leq \frac{1}{C} \int_{0}^{1} f(x) d x \leq \frac{1}{C C_{1}} \int_{0}^{1}\left(\left(x-\frac{1}{2}\right)^{2 n+1}+C_{1}\right) f(x) d x
$$


or equivalently

$$
f^{2}(0) \leq \frac{1}{C^{2} C_{1}^{2}}\left(\int_{0}^{1}\left(\left(x-\frac{1}{2}\right)^{2 n+1}+C_{1}\right) f(x) d x\right)^{2} .
$$

Using (10) and (12) we get (11) and the proof is over.

Example 3.2. If $C=2$ and $C_{1}=\frac{1}{2^{2 n+1}}$ the inequality (9) becomes

$$
f^{4}(0) \leq \frac{1}{4}\left(\int_{0}^{1}\left((2 x-1)^{2 n+1}+1\right) f(x) d x\right)^{2} \int_{0}^{1} f^{\prime 2}(x) d x .
$$

Corollary 3.3. If $g$ is a convex funtction that satisfies the conditions of Th.2.1 then

$$
\left(\int_{0}^{\infty} f(x) d x\right)^{4} \leq \frac{\pi^{2}}{g^{\prime 2}(0)} \int_{0}^{\infty} f^{2}(x) d x \int_{0}^{\infty} g^{2}(x) f^{2}(x) d x
$$

Proof. The proof follows directly from (2) because in this case $\frac{g(x)}{g^{\prime}(0)} \geq x \forall x>0$.

Example 3.4. There are functions that satisfy the conditions of Theorem 2.1 but for wich the proof of the inequality does not follow trivially from Carlson's inequality (2) like in Corrolary (3.3). One of these is

$$
g(x)=\ln \frac{(x+1) \exp x}{x^{2}+1}
$$

Remark 3.5. The Hardy's idea presented in our introduction was very important for the proof of Theorems 2.1 and 2.3. This idea of proof is completely different from the original proof of Carlson, who in fact remarked that (1) does not follow from Hölder's inequality in the following way:

$$
\sum_{n=1}^{\infty} a_{n} \leq\left(\sum_{n=1}^{\infty} a_{n}^{2}\right)^{\frac{1}{4}}\left(\sum_{n=1}^{\infty} n^{2 h} a_{n}^{2}\right)^{\frac{1}{4}}\left(\sum_{n=1}^{\infty} n^{-h}\right)^{\frac{1}{2}}=C(h)\left(\sum_{n=1}^{\infty} a_{n}^{2}\right)^{\frac{1}{4}}\left(\sum_{n=1}^{\infty} n^{2 h} a_{n}^{2}\right)^{\frac{1}{4}}
$$

, because $C(h) \rightarrow \infty$ when $h \rightarrow 1+$.

Remark 3.6. A generalization of (2) in a different direction was recently done in [2]. Some other generalizaions of Carlson's inequalities can be found in the books [11], [1] and the references given there.

\section{References}

[1] Beckenbach, E. F. and Bellman, R., Inequalities, 2nd ed., Springer Verlag, Berlin-New York, 1965. 
[2] Barza, S., Pečarić, J. and Persson, L-E., Carlson type inequalitics, submitted.

[3] Bergh, J., "An optimal reconstruction of sampled signals," J. Math. Anal. Appl., 115(1986), 574-577.

[4] Carlson, F., "Une inégalité, Ark," Mał. Astr. Fysik 25B(1934), 1-5.

[5] Gustavsson, J. and Peetre, J., "Interpolation of Orlicz spaces," Studia Math. 60(1977), 33-59.

[6] Hardy, G. IT. A note on tuo inequalities, J. London Math. Soc. 11(1936), 167-170.

[7] Hardy, G. H., Littlewood, J. E., G. Pólya, G., Incqualitics, Cambriclge Univ. Press, 1952.

[8] Kiellberg, B3., "On some inequalities," C. R. Dixiéme Congrés des Mathematiciens Scandinaves (1946), Jul. Gjellerus Forlag, Copenhagen (1946), 333-340.

[9] Krugijak, N. Ya., Maligranda, L. and Persson, L. E., "^ carlson type incculuality with blocks and interpolation," Studia Math. 104(1993), 161-180.

[10] Maligranda, L. and Persson, L. E., "Inequalities and interpolation," Collect. Math. 44(1993), 183-201.

[11] Mitrinović, D. S., Pečarić, J. E. and Fink, A. M., Inequalities Invoving Finnctions and Their Inegrals and Derivatives, KluwerAcad. Publ., Dordrecht/Boston/London, 1991.

Department of Mathematic, Luleå University, S-97187 Luleå, Swedem.

Faculty of Sciences, University "Lucian Blaga," Ion Ratiu 5-7, 2400 Sibiu, Romania. 